\title{
Observational Characteristics of the Final Stages of Evaporating Primordial Black Holes
}

\section{T. N. Ukwatta}

Space and Remote Sensing (ISR-2), Los Alamos National Laboratory, Los Alamos, NM 87545, USA.

E-mail: tilan@lanl.gov

D. Stump, J. T. Linnemann, S. S. Marinelli, T. Yapici, K. Tollefson

Department of Physics and Astronomy, Michigan State University, East Lansing, MI 48824, USA.

\section{J. H. MacGibbon*}

Department of Physics, University of North Florida, Jacksonville, FL 32224, USA.

\begin{abstract}
Many early universe theories predict the creation of Primordial Black Holes (PBHs). The PBHs could have masses ranging from the Planck mass to $10^{5}$ solar masses or higher depending on the formation scenario. Hawking showed that any Black Hole (BH) has a temperature which is inversely proportional to its mass. Hence a sufficiently small $\mathrm{BH}$ will thermodynamically radiate particles at an ever-increasing rate, continually decreasing its mass and raising its temperature. The final moments of this evaporation phase should be explosive. In this work, we investigate the final few seconds of the $\mathrm{BH}$ burst using the Standard Model of particle physics and calculate the energy dependent burst time profiles in the GeV/TeV range. We use the HAWC (High Altitude Water Cherenkov) observatory as a case study and calculate PBH burst light curves which would be observed by HAWC.
\end{abstract}

The 34th International Cosmic Ray Conference,

30 July- 6 August, 2015

The Hague, The Netherlands

\footnotetext{
* Speaker.
} 


\section{Introduction}

Primordial black holes (PBHs) are black holes created in the early universe. Depending on the formation scenario, the PBHs could have masses ranging from the Planck mass to more than million solar masses [1]. In 1974, Hawking showed by convolving general relativity, thermodynamics and quantum field theory that a Black Hole $(\mathrm{BH})$ has a temperature inversely proportional to its mass and radiates with thermal spectra photons and massive particles [2]. As the $\mathrm{BH}$ emits this radiation, its mass decreases and hence its temperature and emission rate increase. A PBH that formed with an initial mass of $\sim 5.0 \times 10^{11} \mathrm{~kg}$ in the early universe should be expiring today [3] with a burst of high-energy particles, including gamma-rays in the $\mathrm{MeV}$ to $\mathrm{TeV}$ energy range. Thus PBHs are candidate gamma-ray burst (GRB) progenitors [4].

Confirmed detection of a PBH evaporation event would provide valuable insights into many areas of physics including the early universe, high energy particle physics and the convolution of gravitation with thermodynamics. Conversely, non-detection of PBH evaporation events in sky searches would place important limits on models of the early universe.

The properties of the PBH final burst depend on the physics governing the production and decay of high-energy particles. In the Standard Evaporation Model (SEM) which incorporates the Standard Model of particle physics, a BH should directly Hawking-radiate the fundamental Standard Model particles whose Compton wavelengths are of the order of the black hole size [5]. As the $\mathrm{BH}$ evaporates and loses mass over its lifetime, its temperature surpasses the rest mass thresholds of further fundamental particle species. When the temperature exceeds the Quantum Chromodynamics (QCD) confinement scale $(\sim 300 \mathrm{MeV})$, the BH should directly emit quarks and gluons which fragment and hadronize (analogous to jets seen in high-energy collisions in terrestrial accelerators) as they stream away from the $\mathrm{BH}$ into the particles which are stable on astrophysical timescales [5, 3]. Thus according to the SEM, the evaporating black hole will be seen astronomically as a burst of photons, neutrinos, electrons, positrons, protons and anti-protons.

\section{Photons from a PBH Burst}

\subsection{Hawking Radiation}

Hawking showed that a black hole radiates fundamental particle species at an emission rate of $[2,1]$

$$
\frac{d^{2} N}{d E d t}=\frac{\Gamma / 2 \pi \hbar}{e^{x}-(-1)^{2 s}} n_{\mathrm{dof}}
$$

where $s$ is the particle spin and $n_{\text {dof }}$ is the number of degrees of freedom of the particle species (e.g. spin, electric charge, color and flavor). The dimensionless quantity $x$ is defined by

$$
x \equiv \frac{8 \pi G M E}{\hbar c^{3}}=\frac{E}{k T_{B H}}
$$

where $E$ is the energy of the emitted particle, $M$ is the black hole mass, $T_{B H} \propto 1 / M$ is the black hole temperature, and $G, c$, and $\hbar$ are the universal gravitational constant, speed of light, and the reduced Planck constant, respectively. The absorption coefficient $\Gamma$ depends on $M, E$ and $s$. For an 
emitted species of rest mass $m, \Gamma$ at $E \gg m c^{2}$ has the form

$$
\Gamma(M, E, s)=27\left(\frac{x}{8 \pi}\right)^{2} \gamma_{s}(x)
$$

such that $\gamma_{s}(x) \rightarrow 1$ for large $x$.

To calculate the spectrum of the final photon burst from the $\mathrm{PBH}$, two important relations pertaining to the final phase of $\mathrm{BH}$ evaporation are needed. The first relation is the black hole mass $M$ expressed as a function of remaining lifetime. The mass loss rate can be written as [4]

$$
\frac{d M}{d t} \equiv-\frac{\alpha(M)}{M^{2}}
$$

where the factor $\alpha(M)$ incorporates all emitted particle species and degrees of freedom. As the BH evaporates, the value of $M$ is reduced by an amount equal to the total mass-energy of the emitted particles. By conservation of energy,

$$
\frac{d\left(M c^{2}\right)}{d t}=-\sum_{i} \int_{0}^{\infty} \frac{d^{2} N_{i}}{d E d t} E d E
$$

where the summation over $i$ is over all radiated species. Therefore,

$$
\alpha(M)=\frac{M^{2}}{c^{2}} \sum_{i} \int_{0}^{\infty} \frac{d^{2} N_{i}}{d E d t} E d E
$$

Assuming the Standard Model including the top quark, $125 \mathrm{GeV}$ Higgs, and 3 families of massive Majorana neutrinos, we find that the asymptotic value $\alpha_{\mathrm{SM}}$ of $\alpha(M)$ as $M$ decreases is

$$
\alpha_{\mathrm{SM}}=8.40 \times 10^{17} \mathrm{~kg}^{3} \mathrm{~s}^{-1} .
$$

For the current and future generations of high energy gamma-ray observatories, we are interested in bursts generated by black holes of temperature $T_{B H} \gtrsim 1 \mathrm{TeV}$. Thus for $T_{B H} \gtrsim 1 \mathrm{TeV}$ BHs (corresponding to $M \lesssim 10^{7} \mathrm{~kg}$ and a remaining evaporation lifetime of $\tau \lesssim 500 \mathrm{~s}$ ), $\alpha(M) \approx \alpha_{\mathrm{SM}}$ and the $\mathrm{BH}$ mass as a function of remaining evaporation lifetime $\tau$ is

$$
M(\tau) \approx\left(3 \alpha_{\mathrm{SM}} \tau\right)^{1 / 3}=1.36 \times 10^{6}\left(\frac{\tau}{1 s}\right)^{1 / 3} \mathrm{~kg} .
$$

The second relation that we require for the final evaporation phase is the temperature $T_{B H}$ expressed as a function of $\tau$. Combining Equations 2.2 and 2.8, we have

$$
k T_{B H}=\frac{\hbar c^{3}}{8 \pi G M}=7770\left(\frac{1 s}{\tau}\right)^{1 / 3} \mathrm{GeV}
$$

for $k T_{B H} \gtrsim 1 \mathrm{TeV}$.

\subsection{QCD Fragmentation}

According to the SEM, Equation 2.1 describes the direct Hawking radiation of the fundamental particle species of the Standard Model: the leptons, quarks, and the gauge bosons [5, 4]. As they stream away from the BH, these particles will then evolve by Standard Model processes, 
ultimately into the particles which are stable on astrophysical timescales: photons, neutrinos, electrons, positrons, protons, and antiprotons. In particular, quarks and gluons will undergo fragmentation and hadronization into intermediate states which will eventually decay into the astrophysically stable particles.

For application to PBH searches at gamma-ray observatories, we seek the total photon emission rate from the $\mathrm{BH}$. The photon spectrum has several components: (i) the "direct photons" which are directly Hawking radiated by the $\mathrm{BH}$ : this component peaks at a few times $T_{B H}$ and is most important at the highest photon energies at any given $T_{B H}$; (ii) the "fragmentation photons" arising from the fragmentation and hadronization of the directly Hawking radiated quarks and gluons: this component is the dominant source of photons at energies below $T_{B H}$; and (iii) the photons produced by the decays of other Hawking-radiated fundamental particles, e.g. the tau lepton, $W$ and $Z$ gauge bosons, and Higgs bosons: this component is small compared to the component produced by the directly Hawking radiated quarks and gluons and is neglected here. (We note that, because the $W$, $Z$, and Higgs bosons decay predominantly via hadronic channels, the main effect of component (iii) will be to enhance the fragmentation photon component (ii) somewhat.)

\subsection{The Pion Fragmentation Model}

In jet fragmentation and hadronization, most of the photons arise from the decays of $\pi^{0}$ states, which is $\pi^{0}$ decays to $2 \gamma^{\prime}$ 's with a branching fraction $98.8 \%$. We proceed assuming that the QCD fragmentation of quarks and gluons may be approximated entirely by pion production. In our pion fragmentation model, two questions must be addressed: what is the pion distribution generated by the partons (the initial quarks and gluons) and what is the photon spectrum generated by the pion decays?

To answer the first question, we utilize a heuristic fragmentation function

$$
D_{\pi / i}(z)=\frac{15}{16} z^{-3 / 2}(1-z)^{2}
$$

where $z \equiv E_{\pi} / E$ is the energy fraction carried by a pion generated by a parton of energy $E$ [4]. We assume the same form of $D_{\pi / i}(z)$ for all partons and that all of the parton energy goes into pions i.e. the function in Equation 2.10 is normalized such that $\int_{0}^{1} z D_{\pi / i}(z) d z=1$. The instantaneous BH pion production rate per pion energy interval is then

$$
\frac{d^{2} N_{\pi}}{d E_{\pi} d t}=\sum_{i} \int_{m_{\pi} c^{2}}^{\infty} \int_{0}^{1} \frac{d^{2} N_{i}}{d E d t} D_{\pi / i}(z) \delta\left(E_{\pi}-z E\right) d z d E .
$$

\subsection{Photon Flux from Pion Fragmentation}

To answer the second question, we obtain the photon flux from the $\pi^{0} \rightarrow 2 \gamma$ decay of the pion distribution. Because the fragmentation function $D_{\pi / i}(z)$ includes all three pion charge states $\pi^{+}, \pi^{-}$, and $\pi^{0}$ as equal components, and each $\pi^{0}$ decays into two photons, we must multiply by $2 / 3$ to get the $\gamma$ multiplicity. In the $\pi^{0}$ rest frame, the two photons have equal energies, $m_{\pi} c^{2} / 2$ and equal but opposite momenta. In the reference frame of the gamma-ray detector, the energies of the 
two photons are unequal but complementary fractions of the $\pi^{0}$ energy in the detector frame, $E_{\pi}$. We assume that only one of the photons in each pair is detected. ${ }^{1}$

Let $\theta^{\prime}$ be the angle between the momentum of the observed photon in the $\pi^{0}$ rest frame and the momentum of the pion in the detector frame. In the detector frame, the photon energy $E_{\gamma}=$ $\left(E_{\pi} / 2\right)\left(1+\beta \cos \theta^{\prime}\right)$ where the $\pi^{0}$ velocity $\beta=v / c \approx 1$ and $E_{\pi}=m_{\pi} / \sqrt{1-\beta^{2}}$. Because the angular distribution of the photons is isotropic in the $\pi^{0}$ rest frame, the distribution of photon energy in the detector frame is

$$
\frac{d^{2} N_{\gamma}}{d E_{\gamma} d t}=\frac{2}{3} \int_{-1}^{1} \frac{2 \pi d \cos \theta^{\prime}}{4 \pi} \int_{m_{\pi}}^{\infty} \frac{d^{2} N_{\pi}}{d E_{\pi} d t} \delta\left[E_{\gamma}-\left(E_{\pi} / 2\right)\left(1+\beta \cos \theta^{\prime}\right)\right] d E_{\pi} .
$$

\subsection{Parametrization of the Direct and the Fragmentation Contribution}

To further simplify our analysis and subsequent calculations, we parameterize the directly Hawking radiated and the pion fragmentation components of the instantaneous PBH burst photon spectrum using the variable

$$
\xi_{\gamma}=1.287 \times 10^{-4}\left(\frac{E_{\gamma}}{1 \mathrm{GeV}}\right)\left(\frac{1 s}{\tau}\right)^{1 / 3}
$$

as follows:

$$
\left(\frac{d^{2} N_{\gamma}}{d E_{\gamma} d t}\right)_{\text {direct }}=1.13 \times 10^{19}\left(\xi_{\gamma}\right)^{6}\left(\exp \left(\xi_{\gamma}\right)-1\right)^{-1} \times F\left(\xi_{\gamma}\right) \mathrm{GeV}^{-1} \mathrm{~s}^{-1}
$$

where

$$
\begin{aligned}
& F\left(\xi_{\gamma}\right)=1.0 \text { for } \xi_{\gamma} \leq 2 \\
& F\left(\xi_{\gamma}\right)=\exp \left(\left[-0.0962-1.982\left(\ln \xi_{\gamma}-1.908\right)\right]\left[1+\tanh \left[20\left(\ln \xi_{\gamma}-1.908\right)\right]\right]\right) \text { for } \xi_{\gamma}>2
\end{aligned}
$$

and

$$
\begin{aligned}
\left(\frac{d^{2} N_{\gamma}}{d E_{\gamma} d t}\right)_{\text {frag. }} & =6.339 \times 10^{23}\left(\xi_{\gamma}\right)^{-3 / 2}\left[1-\Theta_{S}\left(\xi_{\gamma}-0.3\right)\right] \\
& +1.1367 \times 10^{24} \exp \left(-\xi_{\gamma}\right)\left[\xi_{\gamma}\left(\xi_{\gamma}+1\right)\right]^{-1} \Theta_{S}\left(\xi_{\gamma}-0.3\right) \mathrm{GeV}^{-1} \mathrm{~s}^{-1}
\end{aligned}
$$

where

$$
\Theta_{S}(\lambda)=0.5[1+\tanh (10 \lambda)] .
$$

These parametrizations are valid for energies above $E_{\gamma} \sim 1 \mathrm{GeV}$ and are accurate to $\pm 15 \%$ for $\xi_{\gamma}$ in the range from 0.1 to 5.0 , and to $\pm 3 \%$ for smaller and larger $\xi_{\gamma}$. If greater accuracy is required, we use a table of the ratios of the exact integration of Equation 2.11 using the fragmentation function 2.12 to the parameterized value, to correct the parameterized values.

\footnotetext{
${ }^{1}$ The angle between the 2 photon trajectories in the detector frame will be very small because of the large Lorentz boost. However, if the $\mathrm{BH}$ is at a distance of order 1 parsec from the detector, then only one of the photons from each $\pi^{0}$ decay will hit the detector.
} 


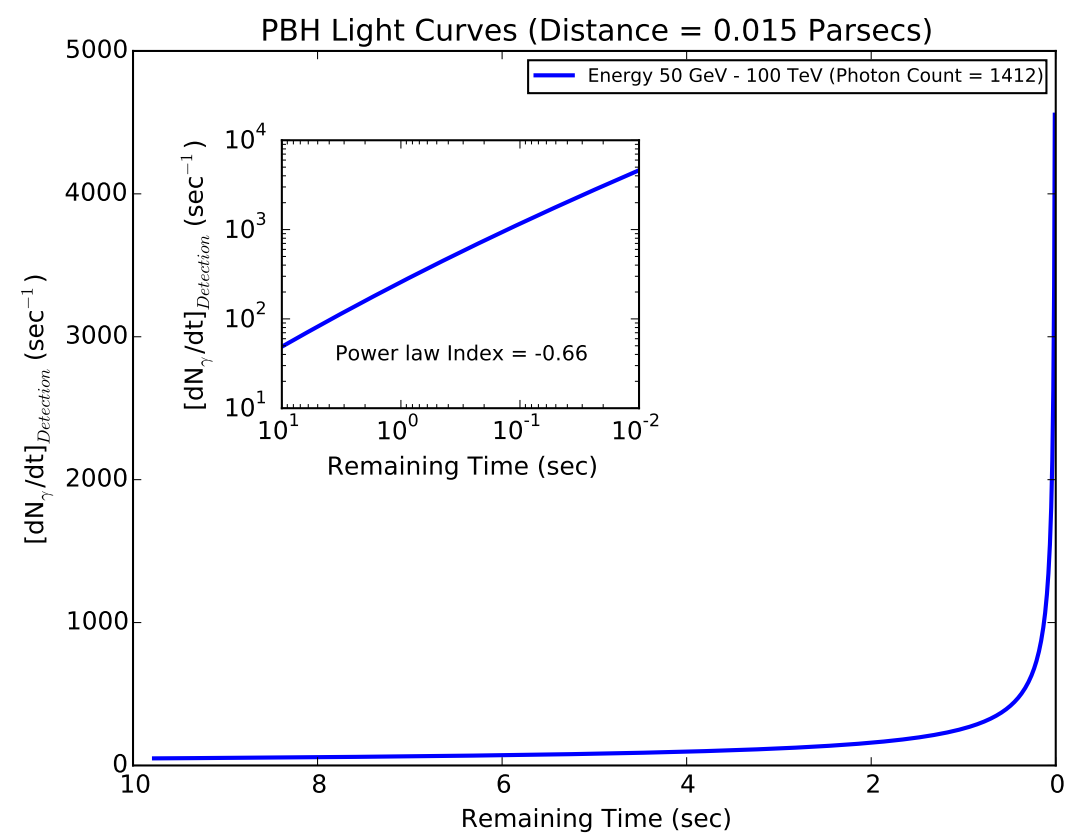

Figure 1: Simulated PBH Burst lightcurve observed by HAWC (at a distance of 0.05 light years) obtained by convolving with the HAWC effective area published in Ref [7]. This shape is also well described by a power law with a index of -0.66 .

\subsection{PBH Burst Light Curve}

To find the time evolution of the PBH burst, we integrate the total instantaneous photon flux $d^{2} N_{\gamma} /\left(d E_{\gamma} d t\right)$ over photon energy $E_{\gamma}$ while retaining the time dependence:

$$
\left[\frac{d N_{\gamma}}{d t}\right]_{\text {Emission }}=\int_{E_{\min }}^{E_{\max }} \frac{d^{2} N_{\gamma}}{d E_{\gamma} d t} d E_{\gamma}
$$

In general $E_{\min }$ and $E_{\max }$ are set by the energy range of the detector. As a case study, we use here the High Altitude Water Cherenkov (HAWC) observatory [6] for which the relevant energy range is $E_{\min }=50 \mathrm{GeV}$ to $E_{\max }=100 \mathrm{TeV}$.

The time profile (the light curve) of the PBH burst at the detector can be calculated as follows:

$$
\left[\frac{d N_{\gamma}}{d t}\right]_{\text {Detection }}=\frac{1}{4 \pi r^{2}} \int_{E_{\min }}^{E_{\max }} A\left(E_{\gamma}\right) \frac{d^{2} N_{\gamma}}{d E_{\gamma} d t} d E_{\gamma}
$$

where $A\left(E_{\gamma}\right)$ is the effective area of the HAWC detector and $r$ is the distance to the PBH. Figure 1 shows the time profile of a PBH burst at a distance of 0.05 light years in the HAWC energy range and for the HAWC effective area published in Ref [7].

It is also interesting to investigate the energy dependence of the PBH burst time profile. In Figure 2, we show $d N_{\gamma} / d t$ calculated using various energy bands in the HAWC energy range. As seen in Figure 2, the low energy bands show similar emission profiles. However, above $E_{\gamma} \sim 10$ 


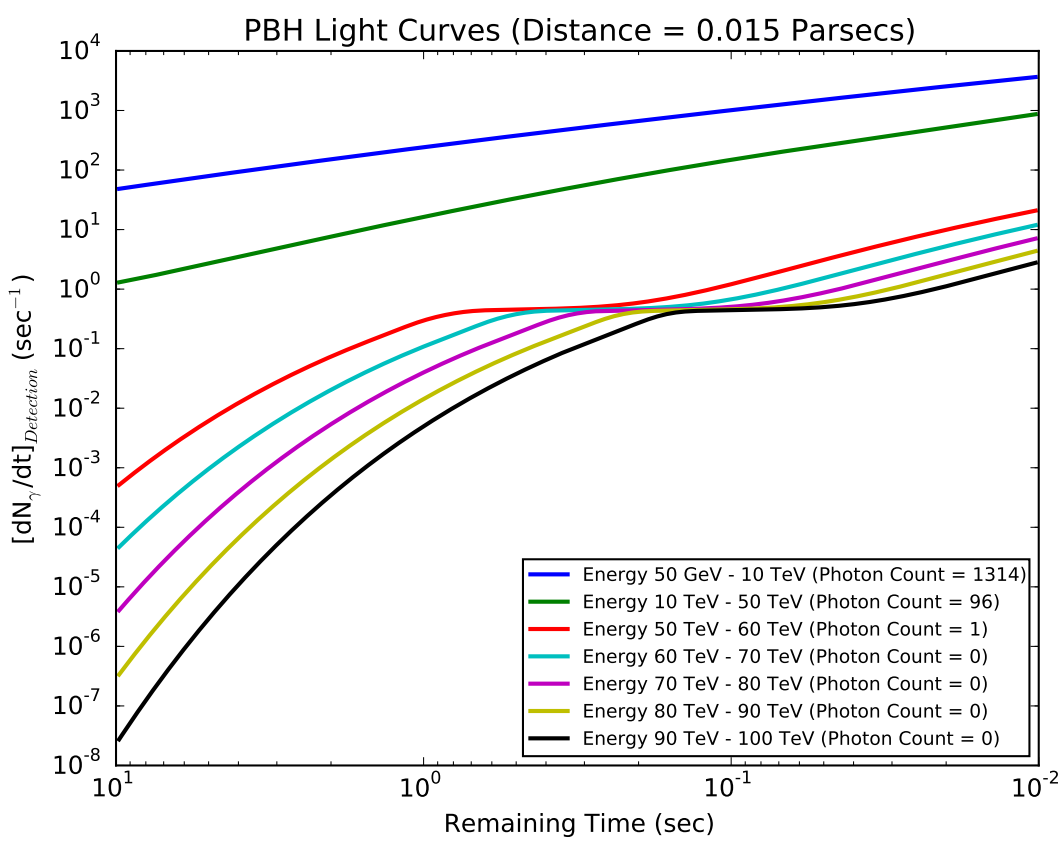

Figure 2: Simulated detection time profiles of a PBH burst at a distance of 0.05 light years observed by HAWC in multiple energy bands. Photon numbers detected in each energy band is shown in the legend.

$\mathrm{TeV}$ the burst emission time profile is energy-dependent and exhibits an inflection around $\tau \sim 0.1$ seconds. This occurs due to the domination of the direct photon component at the highest energies.

\section{Summary and Conclusions}

In this paper, we have briefly reviewed the theoretical framework of the $\mathrm{PBH}$ standard emission model and calculated the PBH burst light curves which would be observed by HAWC. The main findings and conclusions of our paper are:

1. We have developed approximate analytical formulae for the instantaneous PBH spectrum which includes both the directly Hawking radiated photons and the photons arising from the other directly Hawking radiated species.

2. For the first time, we have calculated the PBH burst light curve and studied its energy dependence at a detector.

3. For low energies $\left(E_{\gamma}<10 \mathrm{TeV}\right)$ the light curve profile does not show much evolution with energy and is well described by a power law $\tau^{-0.66}$. However, at high energy the light curve displays significant energy dependence that may be used as an unique signature of PBH bursts. The HAWC observatory is sensitive in this high energy range and potentially can be used to uniquely identify PBH bursts. 


\section{Acknowledgments}

This work was supported by grants from the National Science Foundation (MSU) and Department of Energy (LANL). TNU acknowledges the support of this work by the Laboratory Directed Research \& Development (LDRD) program at LANL. We would also like to thank Alberto Carramiñana of INAOE (National Institute of Astrophysics, Optics and Electronics ) in Mexico and Pat Harding at LANL for useful feedback on the draft of the paper.

\section{References}

[1] B. J. Carr, K. Kohri, Y. Sendouda, and J. Yokoyama, New cosmological constraints on primordial black holes, Phys. Rev. D 81 (May, 2010) 104019, [arXiv:0912.5297].

[2] S. W. Hawking, Black hole explosions?, Nature 248 (Mar., 1974) 30-31.

[3] J. H. MacGibbon, B. J. Carr, and D. N. Page, Do evaporating black holes form photospheres?, Phys. Rev. D 78 (Sept., 2008) 064043, [arXiv: 0709.2380$].$

[4] F. Halzen, E. Zas, J. H. MacGibbon, and T. C. Weekes, Gamma rays and energetic particles from primordial black holes, Nature 353 (Oct., 1991) 807-815.

[5] J. H. MacGibbon and B. R. Webber, Quark- and gluon-jet emission from primordial black holes: The instantaneous spectra, Phys. Rev. D 41 (May, 1990) 3052-3079.

[6] T. N. Ukwatta, D. Stump, J. T. Linnemann, S. S. Marinelli, T. Yapici, K. Tollefson, and J. H. MacGibbon, Sensitivity of HAWC to Primordial Black Hole Bursts, in Proc. 34th ICRC, (The Hague, The Netherlands), August, 2015.

[7] HAWC Collaboration, A. U. Abeysekara, R. Alfaro, C. Alvarez, J. D. Álvarez, R. Arceo, J. C. Arteaga-Velázquez, H. A. Ayala Solares, A. S. Barber, B. M. Baughman, N. Bautista-Elivar, E. Belmont, S. Y. BenZvi, D. Berley, M. Bonilla Rosales, J. Braun, R. A. Caballero-Lopez, K. S. Caballero-Mora, A. Carramiñana, M. Castillo, U. Cotti, J. Cotzomi, E. de la Fuente, C. De León, T. DeYoung, R. Diaz Hernandez, J. C. Díaz-Vélez, B. L. Dingus, M. A. DuVernois, R. W. Ellsworth, A. Fernandez, D. W. Fiorino, N. Fraija, A. Galindo, F. Garfias, L. X. González, M. M. González, J. A. Goodman, V. Grabski, M. Gussert, Z. Hampel-Arias, C. M. Hui, P. Hüntemeyer, A. Imran, A. Iriarte, P. Karn, D. Kieda, G. J. Kunde, A. Lara, R. J. Lauer, W. H. Lee, D. Lennarz, H. León Vargas, E. C. Linares, J. T. Linnemann, M. Longo, R. Luna-GarcIa, A. Marinelli, H. Martinez, O. Martinez, J. Martínez-Castro, J. A. J. Matthews, P. Miranda-Romagnoli, E. Moreno, M. Mostafá, J. Nava, L. Nellen, M. Newbold, R. Noriega-Papaqui, T. Oceguera-Becerra, B. Patricelli, R. Pelayo, E. G. Pérez-Pérez, J. Pretz, C. Rivière, D. Rosa-González, H. Salazar, F. Salesa, F. E. Sanchez, A. Sandoval, E. Santos, M. Schneider, S. Silich, G. Sinnis, A. J. Smith, K. Sparks, R. W. Springer, I. Taboada, P. A. Toale, K. Tollefson, I. Torres, T. N. Ukwatta, L. Villaseñor, T. Weisgarber, S. Westerhoff, I. G. Wisher, J. Wood, G. B. Yodh, P. W. Younk, D. Zaborov, A. Zepeda, and H. Zhou, The HAWC Gamma-Ray Observatory: Dark Matter, Cosmology, and Fundamental Physics, ArXiv e-prints (Sept., 2013) [arXiv:1310.0073]. 